\title{
Are first impressions lasting impressions? An exploration of the generality of the primacy effect in memory for repetitions
}

\author{
JEREMY K. MILLER, DEANNE L. WESTERMAN, and MARIANNE E. LLOYD \\ State University of New York, Binghamton, New York
}

\begin{abstract}
In five experiments, we investigated the primacy effect in memory for repetitions (DiGirolamo \& Hintzman, 1997), the finding that when participants are shown a study list that contains two very similar versions of the same stimulus, memory is biased in the direction of the version that was presented first. In the experiments reported, the generality of the effect was examined by manipulating the orientation and features of the repeated stimuli. The results confirmed that the effect is reliable when stimulus changes affect the accidental properties of the stimulus (properties of the stimulus that give information about distance or angle but do little to aid in identification). However, the effect was not found when changes were made to other aspects of the stimulus. The results suggest that the primacy effect in memory for repetitions is not robust across all stimulus changes and converge with previous findings that have demonstrated that such properties of stimuli as orientation and size are represented differently in memory than are other stimulus characteristics.
\end{abstract}

The beneficial effects of repetition on memory have been well documented (e.g., Ebbinghaus, 1885/1964; for reviews, see Crowder, 1976, and Greene, 1992). Although memory usually improves with repetition of a stimulus, researchers have documented several instances in which increased repetition does not facilitate memory performance. For example, an early study by Nickerson and Adams (1979) showed that participants were unable to properly configure the features of a penny, despite countless repeated exposures to this object.

Recent examples of repetition's failing to facilitate memory performance can be found in studies by Hintzman, Curran, and Oppy (1992) and Hintzman and Curran (1995). In these studies, participants viewed long lists of stimuli, with some stimuli presented as many as 25 times. Later, the participants were given a memory test that included the previously seen items, as well as highly similar lures. The participants were asked to make a frequency judgment for each test item. The results showed that although the participants could report with great accuracy how many times a given stimulus had appeared on a list, repetition did not increase their ability to discriminate a stimulus presented on the list from a highly similar lure.

This research was supported by Grant 1-R03-MH66156-01 from NIMH. The authors thank Colin MacLeod, Gregory DiGirolamo, and two anonymous reviewers for their insightful comments on previous drafts of this article. We also thank Vaibhav Bhatia, Laura Cavallari, Rob Daher, Kristin Dust, Rachael Flora, Melissa Hardy, Tricia Leahy, and Kelly Vaccaro, all of whom assisted in running the participants. Correspondence concerning this article should be addressed to J. K. Miller, Department of Psychology, State University of New York, P.O. Box 6000, Binghamton, NY 13902 (e-mail: jmiller1@binghamton.edu).
Hintzman et al. (1992) termed their finding registration without learning because, although it seemed that the repetition was registered by the participants (as indicated by the increased frequency judgments for repeated items), there was no evidence that the participants learned the details of the stimulus to a degree sufficient to discriminate between the repeated stimulus and a highly similar alternative. For example, the participants were able to report accurately how many times the word frog had appeared on a list; however, they failed to discriminate between the words frog and frogs in a recognition memory test, even when the word frog had appeared many times.

Additional evidence in support of this conclusion was reported in a study by DiGirolamo and Hintzman (1997). In their study, participants studied multiple presentations of simple line drawings of common objects. The study list was constructed so that half of the objects were presented in different left-right orientations. For the objects that were presented in different left-right orientations, either the first presentation or the last presentation was different from all of the others. Immediately following the study phase, the participants were given a forced choice memory test in which both versions of an object from the study list were presented, and the participants' task was to judge whether both versions of the object or only one of the versions had been presented previously. A second experiment, in which stimulus size, rather than orientation, was manipulated was also conducted.

The results of both experiments revealed that errors on the recognition test were biased toward the first presentation of the object. That is, when the participants incorrectly responded that only one version had appeared on the study list, they were more likely to select the version 
that had appeared first. The effect was revealed by comparing the proportion of minority-only responses when the first presentation was different from all subsequent presentations of that object and when the last presentation was different from all preceding versions of that object. A minority-only response was given when a participant reported seeing only the version of the object that was presented once (a minority-only response was incorrect in situations in which both versions of the object had appeared on the study list). DiGirolamo and Hintzman (1997) found that the participants were more likely to give a minority-only response in the first-different condition than in the last-different condition in both of their experiments. In other words, the participants were more likely to remember the version that was presented first, even when it had appeared more recently and more frequently in a different form. DiGirolamo and Hintzman referred to this finding as the primacy effect in memory for repetitions.

The primacy effect described by DiGirolamo and Hintzman (1997) deviates from the traditional incarnation of the primacy effect, insofar as the effect occurs for items that were presented at multiple places throughout the study list, in contrast to the usual finding with items at the beginning of each list. DiGirolamo and Hintzman theorized that the memory system might be weighting the first presentation of the stimulus more heavily than all the subsequent presentations. The authors observed that this explanation is congruent with daily experience, since anyone who has ever had the experience of learning something incorrectly the first time (someone's name, directions for how to set a VCR clock, and so forth) can attest to the fact that first impressions can be very difficult to overcome.

DiGirolamo and Hintzman (1997) proposed three possible explanations for the primacy effect in memory for repetitions. All were similar in that each posited that the primacy effect in memory for repetitions is caused by the way in which repeated stimuli are encoded. One explanation was that the first presentation of the object establishes a representation that is activated by each subsequent presentation. The activation of this initial representation tends to compete with perceptual information about the current stimulus that would indicate that the attributes of the object have changed. The result of this competition is that attributes of the first presentation of an object tend to eclipse the currently perceived attributes, resulting in a perceptual representation that resembles the first presentation of that object more than it resembles the current presentation. The second proposed explanation was that changed attributes of the stimuli are perceived correctly but that the encoding into memory that follows is biased by previous presentations. The resulting memory trace is stored as a representation that shares attributes of previous presentations of the item. The third type of explanation was that familiar stimuli are given less attention than are novel stimuli; therefore, the first presentation is remembered better than subsequent presentations.

The notion that the first and subsequent presentations of a stimulus are processed differently is supported by a great deal of evidence from both the cognitive and the cognitive-neuroscience traditions. For example, eventrelated potential (ERP) studies have shown that the ERPs of repeated presentations are significantly different from those of first presentations of line drawings of objects (Rugg, Soardi, \& Doyle, 1995). Research with positron emission tomography (PET) has shown that cortical and subcortical areas in the temporal lobes respond more actively to novel stimuli than to comparable familiar stimuli (Tulving, Markowitsch, Kapur, Habib, \& Houle, 1994). Also, research in which eye-tracking technology has been used has shown that when participants are exposed to repeated presentations of a scene, the participants make fewer fixations and sample fewer regions of the stimulus (e.g., Althoff \& Cohen, 1999). All of these findings provide converging evidence for the idea that the encoding of repeated stimuli may be impoverished when compared with the encoding carried out on novel stimuli.

One way to gauge the extent to which a stimulus is being encoded is to measure the time that participants spend looking at that stimulus (e.g., Rao \& Proctor, 1984). Therefore, in contrast to previous work in which the primacy effect in memory for repetitions has been examined (DiGirolamo \& Hintzman, 1997), in the present experiments, a participant-paced study list was used. By measuring the amount of time that participants spent looking at a given stimulus, we could obtain a rough index of the amount of encoding that was devoted to a particular stimulus, which would allow us to make judgments about the extent to which impoverished encoding contributes to the primacy effect in memory for repetitions.

A second purpose of these experiments was to test the generality of the effect across different encoding conditions and different types of stimulus changes across repetitions. It was also an aim of the experiments to determine whether the effect would persist when an attempt was made to equalize the encoding resources devoted to the initial and subsequent presentations of an object (Experiment 2). Experiments were also conducted to determine the generality of the primacy effect when different dimensions of the stimuli (e.g., color and object features) were changed between the first and subsequent presentations (Experiments 3, 4, and 5). The rationale for examining the generality of the primacy effect across different stimulus changes is that there are several findings that suggest that objects that vary only in their left-right orientation and size (the manipulations used in the 1997 DiGirolamo \& Hintzman study) may be treated as the same object by the cognitive system (Biederman \& Gerhardstein, 1993; Ellis, Allport, Humphreys, \& Collis, 1989; Kanwisher, Yin, \& Wojciulik, 1999). Therefore, this effect may be more likely to occur with size and orientation changes than with other changes to objects.

\section{EXPERIMENT 1}

DiGirolamo and Hintzman (1997) found that when participants were shown multiple presentations of two different versions of a stimulus - one "normal" version 
and one mirror reversal (rotated $180^{\circ}$ ) - they were more likely to report seeing the version that was presented first, rather than the version that was presented more recently. DiGirolamo and Hintzman theorized that as a stimulus becomes more familiar, the amount of attention that it is given may be attenuated, and as a consequence, learning about the stimulus may be impaired. DiGirolamo and Hintzman further theorized that a strong familiarity signal triggered by a repeated item could modulate the manner in which this stimulus is encoded (e.g., the repetition may be noted, but further information regarding the stimulus, such as changes in orientation or size, is not stored). Experiment 1 was designed to replicate the findings of DiGirolamo and Hintzman, confirming that the effect can be found when slightly different methods and procedures are used.

Experiment 1 also was designed to provide a preliminary test of the explanation that the primacy effect in memory for repetitions occurs because more attention is paid to novel stimuli than to familiar stimuli. With this goal in mind, we changed the study phase from an experimenterpaced presentation of the study list to a participant-paced presentation of the study list. In this experiment, the participants could move at their own pace through the list, spending as much time on each presentation of each object as they felt that they needed. The amount of time that each presentation was studied was recorded. Although study time is affected by a number of variables (Dunlosky \& Thiede, 1998), this measure should provide us with a rough method for quantifying how much attention is paid to each presentation of the object (Rao \& Proctor, 1984; Shaughnessy, Zimmerman, \& Underwood, 1972; Zimmerman, 1975).

\section{Method}

Participants. Thirty Binghamton University students were participants and received partial credit toward a course requirement. The participants were tested individually.

Design and Materials. The design and materials in Experiment 1 were closely modeled after those used by DiGirolamo and Hintzman (1997). Bitmaps of 84 objects were selected from a pool developed by Snodgrass and Vanderwart (1980). The objects were selected for asymmetry, to facilitate the creation of distinctive mirrorreversed stimuli. Two versions of each of the objects were created; one version was at its normal orientation, and one version was a mirror reversal of the object, which consisted of the object flipped $180^{\circ}$ horizontally. The objects were then divided randomly into two groups of 12 to serve as buffers and three groups of 20 for use as critical items.

The study list consisted of 420 presentations of these objects. The objects were presented one at a time in the center of the screen, and the participants moved from one item to the next by pressing the space bar. The 420 presentations were divided into five blocks. The first and last blocks were buffer blocks and consisted of objects assigned to the two buffer groups. These buffer objects were presented five times each in a random order within the buffer block, so that each participant saw the buffers in a different random order. The middle three blocks consisted of the critical items, which would be included on a later recognition test. Critical items were further randomly divided into three categories: first different, last different, and all same. Of the 60 critical objects, 20 randomly selected objects appeared in each of the critical blocks. All critical blocks were further divided into five segments, and each of the five presentations of each critical object was assigned to one of the five segments within a block. Therefore, each critical block consisted of 100 presentations of the objects, 20 for each segment. Within a segment, the items were presented in a random order.

For critical items assigned to the first-different condition, the presentation of the object in the first segment was displayed at a reversed orientation from the presentations shown in Segments 2-5. For example, if a picture of a camel was shown facing to the right in Segment 1, it was shown facing to the left in Segments 2, 3, 4, and 5. For items assigned to the last-different condition, the presentations in Segments 1-4 were shown at one orientation, and the presentation in Segment 5 was shown at the reverse orientation. Items assigned to the all-same and buffer conditions were shown at the same orientation in all the segments.

To control for serial position, the last segment of Block 2 was mixed with the first segment of Block 3, and the last segment of Block 3 was mixed with the first segment of Block 4 . Separate counterbalanced lists were created so that each object appeared equally often in each condition and each orientation. So a given object appeared equally often in its forward-facing and mirror-reversed orientations, as well as equally often in each condition (first different, last different, and all same).

Procedure. This experiment closely adhered to the procedures outlined by DiGirolamo and Hintzman (1997). In the study phase, the participants were exposed to the list as just described. The participants were instructed to try to remember each object on the list, in preparation for a memory test. The exact nature of the memory test was not disclosed. The participants were told that many of the stimuli would be repeated and that they should try to pay close attention to each presentation of each picture. All the stimuli were presented on 17-in. color monitors.

The participants were seated approximately $76 \mathrm{~cm}$ from the monitor. Test stimuli showed both versions of the critical object, with one version appearing in a red box $(10.16 \times 10.16 \mathrm{~cm})$ on the left-hand side of the screen and the mirror-reversed version of the object appearing in a purple box $(10.16 \times 10.16 \mathrm{~cm})$ on the right-hand side of the screen. Below the two boxes was a third, orange box $(8.9 \times$ $2.2 \mathrm{~cm}$ ) with the word both printed in it. The participants were instructed to press a red key on the keyboard in front of them if they thought that they had seen only the version of the object in the red box, to press a purple key if they thought that they had seen only the version of the object in the purple box, and to press an orange key if they thought they had seen both versions of the object. The test was self-paced; as soon as a participant provided an answer to one test item, the next test item appeared on the screen.

\section{Results and Discussion}

For this experiment and all the others reported in this article, data were collected for all trials and for serially balanced trials only, as previously was done by DiGirolamo and Hintzman (1997). Therefore, two sets of data were collected from each participant — one that consisted of a participant's performance on all trials and one that consisted only of performance on serially balanced trials. Serially balanced trials are trials in which list position is equated, in that critical items from each experimental condition occur at roughly similar serial positions during the presentation of the list. Analyses based on serially balanced trials revealed results similar to those when all the trials were included in the analyses. Therefore, only the results of the analyses of all the trials are reported here. The criterion for significance used for all of the experiments was .05 . 
The comparison of central interest in this experiment is between the proportion of minority-only responses given to test objects in the first-different condition (the proportion of times that the participant chose the object that had been presented only once and that object had been presented first) and the proportion of minority-only responses given to test items in the last-different condition (the percentage of times that the participant chose the object that had been presented only once and that object had been presented last of the five repetitions). To make this comparison, response data for each participant for each of the trial types were converted into the proportion of trials on which the participant had responded with the minority-only, the majority-only, or the both response. A summary of the results can be found in Table 1 .

A 3 (type: all same, first different, or last different) $\times$ 3 (response: majority, minority, or both) repeated measures analysis of variance (ANOVA) was conducted on these data, revealing a main effect of response $[F(2,58)=$ $\left.11.74, M S_{\mathrm{e}}=0.06\right]$. This effect reflects the fact that the participants were more likely to make a both judgment than to choose either of the other two response options. A response $\times$ type interaction $\left[F(4,116)=11.51, M S_{\mathrm{e}}=\right.$ $0.03]$ was also obtained. This interaction shows that the participants responded differently across the three experimental conditions. Planned comparisons revealed that the participants were more likely to choose the minorityonly response in the first-different condition than in the last-different condition, revealing a primacy effect in memory for repetitions $[t(29)=3.84, S E=0.03]$.

To determine whether the participants spent more time looking at the first presentation of each stimulus during the encoding phase, the amount of time (in milliseconds) that the participants studied each object during the encoding phase was analyzed. A one-way ANOVA conducted to compare study time for each of the five presentations of a stimulus was significant $[F(4,116)=$ $12.99, M S_{\mathrm{e}}=23,143$ ], reflecting the fact that the participants tended to look longer at earlier presentations of an object. Pairwise comparisons revealed significant differences between study times for each of the five presentations of each of the objects, except for the second and fifth presentations, which did not vary significantly from each other. Study time data are presented in Table 2. In general, the participants studied the first presentation of the object the longest, with study time decreasing until the final presentation, which shows a marked increase in looking time. This increase could be an artifact of the manner in which the list was constructed, since the last segment of each block of trials was mixed with the first segment of the subsequent block. The increase may reflect a general slowing down in processing as the participants noticed that objects that were not repetitions were starting to appear again.

The looking time for each critical condition within a segment was also examined. In this way, looking time for items in the first-different and last-different conditions could be compared with looking time in the all-same condition. These data were subjected to a two-way repeated measures ANOVA, with condition (three levels: all same, first different, or last different) and presentation (five levels: List Segments 1-5) as within-subjects factors. The results of the ANOVA revealed a significant effect of list position $\left[F(4,128)=13.1, M S_{\mathrm{e}}=639,938\right]$, indicating

Table 1

Experiments 1 and 2: Proportion of Response Types for All Trials

\begin{tabular}{|c|c|c|c|c|c|c|c|}
\hline \multirow[b]{3}{*}{ Experiment } & \multirow[b]{3}{*}{ Condition } & \multicolumn{6}{|c|}{ Response } \\
\hline & & \multicolumn{2}{|c|}{ Minority Only } & \multicolumn{2}{|c|}{ Majority Only } & \multicolumn{2}{|c|}{ Both } \\
\hline & & $M$ & $S D$ & $M$ & $S D$ & $M$ & $S D$ \\
\hline \multirow[t]{3}{*}{1} & All same & .20 & .12 & .51 & .15 & .29 & .15 \\
\hline & First different & .24 & .13 & .36 & .17 & .40 & .19 \\
\hline & Last different & .17 & .14 & .35 & .16 & .48 & .19 \\
\hline \multirow[t]{3}{*}{2} & All same & .15 & .08 & .56 & .14 & .28 & .13 \\
\hline & First different & .16 & .14 & .23 & .15 & .61 & .21 \\
\hline & Last different & .09 & .11 & .36 & .19 & .55 & .21 \\
\hline
\end{tabular}

Table 2

Experiments 1-5: Mean Study Time and Standard Deviations (in Milliseconds) by Presentation

\begin{tabular}{|c|c|c|c|c|c|c|c|c|c|c|}
\hline \multirow[b]{3}{*}{ Experiment } & \multicolumn{10}{|c|}{ Presentation } \\
\hline & \multicolumn{2}{|c|}{1} & \multicolumn{2}{|c|}{2} & \multicolumn{2}{|c|}{3} & \multicolumn{2}{|c|}{4} & \multicolumn{2}{|c|}{5} \\
\hline & $M$ & $S D$ & $M$ & $S D$ & $M$ & $S D$ & $M$ & $S D$ & $M$ & $S D$ \\
\hline 1 & 1,715 & 155 & 1,185 & 116 & 985 & 93 & 915 & 98 & 1,100 & 114 \\
\hline 2 & 1,287 & 54 & 932 & 33 & 817 & 34 & 804 & 38 & 825 & 35 \\
\hline 3 & 1,480 & 93 & 1,144 & 70 & 983 & 55 & 898 & 51 & 991 & 50 \\
\hline 4 & 1,577 & 145 & 1,330 & 124 & 1,133 & 93 & 1,108 & 93 & 1,218 & 108 \\
\hline 5 & 1,361 & 117 & 1,026 & 87 & 930 & 80 & 801 & 76 & 900 & 83 \\
\hline
\end{tabular}


that the participants' looking time was different depending on the list segment they were viewing. No main effect of condition was found $\left[F(2,64)=.87, M S_{\mathrm{e}}=43,863\right.$, $p=.42]$. Generally, the participants studied the first presentation of an object longest, with subsequent presentations garnering less and less study time, until the last presentation, which was studied a bit longer (probably an artifact of list construction, as was mentioned above), and this pattern did not vary with experimental condition. Therefore, the participants did not change their study strategy as a function of experimental condition. Similar analyses were also performed for all the subsequent experiments, but because the effects of experimental condition were null in each case, these analyses are not presented for Experiments 2-5. ${ }^{1}$

To summarize, Experiment 1 replicated the findings of DiGirolamo and Hintzman (1997). The participants reported seeing only the minority presentation of an object more often in the first-different condition than in the last-different condition. The results also show that the participants spent more time studying the first presentation of an object than studying later presentations. This finding suggests that participants attend more to the first presentation of an object and lends support to the idea that they devote more encoding resources to the initial presentation of an object than to subsequent presentations. In Experiment 2, this idea was further examined by attempting to equalize the types of encoding processes that were carried out for first and subsequent presentations of the same objects.

\section{EXPERIMENT 2}

The primacy effect in memory for repetitions bears some resemblance to the experimental finding known as change blindness (O’Regan, Rensink, \& Clark, 1999; Simons \& Levin, 1998; see Simons, 2000, for a review). Change blindness is the finding that participants have difficulty detecting a change to a stimulus if the change occurs during a break in the processing of that stimulus. The primacy effect in memory for repetitions resembles change blindness in that participants seem to fail to update their representation of an object upon reencountering the item in a changed perceptual form (e.g., a new orientation) after a break in processing. Researchers interested in change blindness have used the phenomenon to argue that the mental representation of visually perceived objects is quite sparse (Levin, Simons, Angelone, \& Chabris, 2002; Simons \& Levin, 1998) and that, as a result, minor inconsistencies across visual scenes are not detected. It is possible that the primacy effect in memory for repetitions is also a product of the sparseness of this mental representation. That is, participants may not always notice the change in objects across repetitions.

Experiment 2 was an attempt to ensure that the participants attended to spatial orientation information by asking them to make a judgment about the orientation of each stimulus as it was presented on the study list. This type of study list manipulation has been used previously by Srinivas (1996). In this way, we can be assured that the orientations of the repeated stimuli were noticed at the time that they were presented. In addition, this manipulation should have increased the salience of orientation information for the participants, motivating the participants to place special emphasis on the encoding of orientation changes. If the primacy effect in memory for repetitions occurs because participants do not notice the changing orientation of the objects, asking participants to attend to orientation information during encoding should attenuate the effect.

\section{Method}

Participants. Forty-nine Binghamton University students participated toward partial completion of a course requirement.

Design and Materials. Experiment 2 was designed identically to Experiment 1, with one exception: During the presentation of the study list, the participants were asked to make a judgment regarding whether they believed that each stimulus was facing to the right or to the left. The phrase is this object facing right or left? appeared in the lower portion of the screen, 2 in. below each object. For some of the objects, the answer to this question was subjective, since not all of the objects were easily classified into right- and left-facing categories. The participants were encouraged to give the answer that they felt was best when the answer was not obvious. The participants were not told to memorize the object orientations but, rather, to simply indicate whether the object was facing to the right or to the left.

Procedure. The procedure was identical to that in Experiment 1, except for the addition of the instructions regarding the left-right judgment task. The participants were instructed to press a key marked right if they thought that the object was facing to the right and to press a key marked left if they thought that the object was facing to the left. The participants had as much time as they wanted to make their responses, at which point the next item on the list was presented.

\section{Results and Discussion}

Once again, the comparison of central interest in this experiment was between the proportion of minority-only responses in the first-different condition and the proportion of minority-only responses in the last-different condition. The responses were divided into minority only, majority only, and both, and the proportions of each of these response types in each of the conditions were calculated. The results are summarized in Table 1. A 3 (type: all same, first different, or last different) $\times 3$ (response: majority, minority, or both) repeated measures ANOVA was conducted on the data, revealing a main effect of response $\left[F(2,96)=80.4, M S_{\mathrm{e}}=0.05\right]$. This effect shows that the participants were more likely to make a both judgment than to choose either of the other two response options. A significant response $\times$ type interaction $\left[F(4,192)=60.32, M S_{\mathrm{e}}=0.03\right]$ was also obtained, indicating that the combination of the three experimental conditions had an influence on the way that the participants responded. A $t$ test revealed a significant difference between the first-different and the last-different conditions in the minority-only response category, revealing a primacy effect in memory for repetitions $\left[t(48)=6.14, M S_{\mathrm{e}}=0.02\right]$. 
Experiment 2 was designed to ensure that the participants focused on and encoded the attribute that would allow them to discriminate between differing versions of the stimuli during the forced choice recognition test. This manipulation may also have served to equalize the amount of attention given to each presentation of the repeated objects (or at least the amount of attention given to the most relevant aspects of the stimulus for the test). Despite these efforts, a primacy effect for repetitions was found, suggesting that the effect failed to occur because the participants were blind to the changed attribute (i.e., orientation change) across trials.

\section{EXPERIMENT 3}

So far, we have focused on differences in the amount of attention given to the first and subsequent presentations of an object as a possible mechanism responsible for the primacy effect in memory for repetitions. Another factor that may contribute to primacy effects such as those observed in Experiments 1 and 2 is the type of stimulus changes that are used to demonstrate this effect. DiGirolamo and Hintzman (1997) changed their stimuli by presenting mirror reversals or by changing the size of the stimuli. There are numerous studies that suggest that participants may have difficulty distinguishing between stimuli that vary only in orientation and size. For example, Bartlett, Gernsbacher, and Till (1987) have documented that participants have difficulty distinguishing stimuli from their mirror reversals.

In addition, a study by Kanwisher et al. (1999, Experiment 2) provides evidence for the idea that the visual codes involved in object identification are invariant with respect to size and orientation. In their experiments, participants saw pictures of objects presented in rapid succession. Within the stream of objects, there was a direct repetition of an object, a picture of the same object in a different orientation, or a picture of the same object in a different size. The participants failed to notice repeated objects within the stream (repetition blindness) in each of these conditions, a finding that supports the idea of size- and viewpoint-invariant object representations.

If participants' representations of objects are size and viewpoint invariant, it may have an effect on the participants' memory for these items. The role of orientation in memory has been the subject of empirical enquiry. Generally, changes in stimulus orientation between study and test have an adverse effect on memory (Srinivas, 1995, 1996), but this is not always the case. For example, in a study by Madigan and Rouse (1974), participants had difficulty discriminating between previously observed scenes and the same scene in a reversed orientation. In addition, research by Srinivas $(1995,1996)$ has shown that under some circumstances, stimulus factors such as leftright orientation do not influence performance on perceptual implicit memory tests. Findings such as this led Roediger and Srinivas (1993) to suggest that the perceptual system is less sensitive to features that reflect only $a c$ - cidental properties of objects (properties, such as leftright orientation and object size, that provide little information that aids in the process of object identification).

Changes in the accidental properties of stimuli across repetitions in the study list may lead to a primacy effect in memory for repetitions, because the cognitive system does not encode the changed attribute of the stimuli. It is possible that upon encountering a mirror-reversed version of a stimulus that was previously studied, the version of the object that was originally studied is reactivated (as opposed to the formation of a new separate representation of the object in its mirror-reversed form) and, consequently, this is the version recognized at test. This type of account of the primacy effect in memory for repetitions would fall in line with previous findings from the registration-without-learning literature, in which repetitions are registered but do not increase the ability to discriminate between similar stimuli.

The primacy effect in memory for repetitions has also been found when object size (and not just left-right orientation) was changed across repetitions (DiGirolamo \& Hintzman, 1997). However, object size is similar to object orientation in that both can be seen as manipulations of the object's position in three-dimensional (3-D) space (for changes in size, we can picture the object as having moved further away from or closer to us within the visual field). Work by Schacter, Cooper, and collaborators (Cooper, Schacter, Ballasteros, \& Moore, 1992; Schacter, Cooper, \& Delaney, 1990) suggests that retinal size and left-right orientation belong to a class of object properties that are less effective as cues for recognition, due to the fact that they are not encoded as part of a structural description of the object. The structural description outlined by Schacter and Cooper is a description of the global relations among the components of an object. In Experiment 3, we set out to answer the question of whether the effect will occur if the identity of the object is changed, as opposed to the object's position in 3-D space.

In Experiment 3, some of the features of the objects were manipulated across repetitions; this manipulation was used because participants may be less likely to regard two objects that are very similar as the same object when they have slightly different features, as compared with when they are presented at different orientations. Alternatively, the primacy effect may be general to any minor changes in the stimulus. If that is the case, it should persist with changes other than orientation and size changes.

\section{Method}

Participants. Fifty-three Binghamton University students participated to partially fulfill a course requirement.

Materials and Procedure. New stimulus lists were created for Experiment 3. The lists were constructed in the same format and length as the lists in Experiment 2, with the original stimuli again drawn from Snodgrass and Vanderwart (1980). An alternate version of each of the selected stimuli was prepared by modifying the original stimulus slightly. For instance, a bitmap depicting a butterfly was altered so that the pattern on the wings of the butterfly was different, and a bitmap depicting a camel was changed from one 
hump to two. The study and recognition test procedures were the same as those in Experiment 1.

\section{Results and Discussion}

The data were divided into three response categories: minority only, majority only, and both. The proportion of responses in each of these categories for each condition is reported in Table 1. A 3 (type: all same, first different, or last different) $\times 3$ (response: majority, minority, or both) repeated measures ANOVA was conducted on these data. This analysis revealed a main effect of response $\left[F(2,104)=24.33, M S_{\mathrm{e}}=0.07\right]$, showing that the participants were more likely to make a both judgment than to choose either of the other two response options. A response $\times$ type interaction $[F(4,208)=18.61$, $\left.M S_{\mathrm{e}}=0.02\right]$ was again obtained and was interpreted as in the previous experiments. Planned comparisons in this experiment revealed that the participants were not more likely to choose the minority-only response in the firstdifferent condition than in the last-different condition, in contrast with the results of the previous experiments reported here. A planned comparison $t$ test revealed no significant primacy effect in this experiment, despite the presence of a slight numerical trend in the direction of the effect $\left[t(52)=1.67, M S_{\mathrm{e}}=0.02\right]$. The proportion of the participants who reported seeing only the minorityonly response in the first-different condition was .25 , whereas the proportion of the participants reporting seeing only the minority-only version of the stimulus in the last-different condition was .22. In Experiment 3, almost twice as many participants were used as in the previous experiments in which the effect was found, so it seems unlikely that insufficient power is solely responsible for the null effect.

Study time data were once again analyzed in this experiment. The average times that each participant spent looking at an object as a function of its order of presentation were compared. A one-way ANOVA indicated that there were differences between the participants' study times for each presentation of an object $[F(4,208)=$ $\left.60.59, M S_{\mathrm{e}}=80,580\right]$. Pairwise comparisons revealed that each of the five presentation positions was different from each other, except the third and fifth presentations, which did not vary significantly from each other. It is interesting to note that the looking time data of this experiment showed a pattern similar to those in Experiments 1 and 2, despite the fact that no primacy effect was observed in this experiment.

In summary, the results of Experiment 3 revealed no significant primacy effect in memory for repetitions, although a nonsignificant numerical trend was observed. The finding of a null effect supports the notion that the effect may be specific to orientation and size changes, since the effect did not emerge when object features were changed. The fact that study time measures showed a pattern similar to those found in previous experiments suggests that the participants had the same approach to studying the objects: They spent more time studying the first presentation than studying subsequent presentations. Nevertheless, a primacy effect in recognition of the objects did not emerge. Experiment 4 was an attempt to determine whether another type of stimulus change would result in a primacy effect in recognition of similar objects.

\section{EXPERIMENT 4}

The results of Experiment 3 suggest that the primacy effect in memory for repetitions may be specific to orientation-based stimulus manipulations. In Experiment 4 , the primacy effect in memory for repetitions was investigated further with changes other than size and orientation changes. In Experiment 4, the object manipulation was a color change. This experiment helped to explore further the generality of the primacy effect in memory for repetitions and to determine whether the effect occurs when object changes other than size and orientation are made.

\section{Method}

Participants. Forty-two participants participated in the experiment in exchange for partial fulfillment of a course requirement.

Design and Materials. The method used in this experiment was identical to that in Experiment 3, except that instead of the altered object stimulus set used in Experiment 3, a new stimulus set consisting of objects of different colors was used. Two versions of each object were prepared, a black version and a green version. The green version of the stimuli consisted of the same bitmaps as those used in the previous experiments; however, the lines making up the stimuli were colored green instead of black. Green was chosen for the alternative color since it was dark enough that the objects were still clearly visible and yet bright enough to provide an obvious contrast with the black stimuli.

\section{Results and Discussion}

The data from Experiment 4 were analyzed in the same manner as the data from the previous three experiments. Proportions of responses in each response category by condition are reported in Table 3 . The data of 2 participants who gave more minority-only responses than majority-only responses in the all-same condition were completely deleted from the analysis, following the conventions set by DiGirolamo and Hintzman (1997).

The pattern of results observed in this experiment was very similar to that in Experiment 3. A 3 (type: all same, first different, or last different) $\times 3$ (response: majority, minority, or both) repeated measures ANOVA was conducted on the data. This analysis revealed a main effect of response $\left[F(2,82)=93.22, M S_{\mathrm{e}}=0.07\right]$, reflecting the fact that the participants were more likely to make a both judgment than to choose either of the other two response options. A response $\times$ type interaction $[F(4,164)=$ $\left.15.84, M S_{\mathrm{e}}=0.01\right]$ was again obtained and was interpreted as in the previous experiments. Planned comparisons revealed that the participants were not more likely to choose the minority-only response in the first-different condition than in the last-different condition, in contrast 
Table 3

Experiments 3-5: Proportion of Response Types for All Trials

\begin{tabular}{|c|c|c|c|c|c|c|c|}
\hline \multirow[b]{3}{*}{ Experiment } & \multirow[b]{3}{*}{ Condition } & \multicolumn{6}{|c|}{ Response } \\
\hline & & \multicolumn{2}{|c|}{ Minority Only } & \multicolumn{2}{|c|}{ Majority Only } & \multicolumn{2}{|c|}{ Both } \\
\hline & & $M$ & $S D$ & $M$ & $S D$ & $M$ & $S D$ \\
\hline \multirow[t]{3}{*}{3} & All same & .19 & .09 & .51 & .14 & .30 & .15 \\
\hline & First different & .25 & .13 & .33 & .15 & .42 & .23 \\
\hline & Last different & .22 & .12 & .36 & .18 & .42 & .23 \\
\hline \multirow[t]{3}{*}{4} & All same & .12 & .10 & .37 & .19 & .51 & .12 \\
\hline & First different & .16 & .11 & .26 & .13 & .57 & .17 \\
\hline & Last different & .12 & .10 & .26 & .16 & .61 & .19 \\
\hline \multirow[t]{3}{*}{5} & All same & .12 & .07 & .45 & .14 & .42 & .12 \\
\hline & First different & .17 & .12 & .25 & .14 & .57 & .11 \\
\hline & Last different & .15 & .09 & .29 & .14 & .55 & .10 \\
\hline
\end{tabular}

with the results of the previous experiments reported here. There was no significant primacy effect in this experiment, despite the presence of a slight numerical trend in that direction $\left[t(41)=1.05, M S_{\mathrm{e}}=0.03\right]$. The proportion of the participants who reported seeing only the minority-only version of the stimulus was .16 in the first-different condition and .12 in the last-different condition. No significant primacy effect in memory for repetitions was observed.

The average time that each participant spent looking at an object depending on its order of presentation was again compared. A one-way ANOVA indicated that there were differences in the participants' study times for each presentation of an object $\left[F(4,164)=15.2, M S_{\mathrm{e}}=74,130\right]$. Pairwise comparisons revealed that Presentations 1 and 2 and Presentations 2 and 3 were different from each other but that Presentations 3 and 4 and Presentations 4 and 5 were not different from each other.

As in the previous experiment, in which the features of the objects were manipulated, no significant primacy effect in memory for repetitions was observed in this experiment. In conjunction, Experiments 3 and 4 suggest that changing only the features of an object eliminates the primacy effect in memory for repetitions. However, it is possible that the null primacy effects in Experiments 3 and 4 occurred because the participants were not registering the changed objects as repetitions, despite their obvious physical similarities. It may be that there is something critical about the participant's perceiving the object as a repetition, rather than as a similar member of the same category. If participants initially identify the object as a repetition, they may be disinclined to fully process the stimulus, whereas if they perceive the stimulus as a similar but novel exemplar of a previously seen category of stimuli (e.g., butterflies), they may process the stimulus carefully, in anticipation of having to discriminate the two similar category exemplars on the memory test. Indeed, it is easy to accept that these objects may not be perceived as repetitions, due to the fact that critical features of the object have changed in Experiments 3 and 4, and few would look at two photos of a butterfly's wings, one with one pattern and one with another, and say that they have seen the same butterfly. Experiment 5 was an attempt to examine this issue more closely by keeping the features of the objects intact across presentations. Experiment 5 returns to a manipulation of object orientation in order to further investigate this effect.

\section{EXPERIMENT 5}

Given evidence from previous research that shows that left-right reflection and stimulus size may be poor cues to recognition (Bartlett et al., 1987; Cooper et al., 1992), it is possible that the orientation manipulations used in the previous experiments were not salient enough to encourage the participants to process orientation-manipulated objects as separate objects (despite instructions to do so in some cases [Experiment 2]). Experiment 5 was designed with the idea that perhaps a more salient orientation change would encourage the participants to do this. The methods used in Experiment 5 was the same as those reported in the previous experiments, with the exception that objects were now inverted instead of mirror reversed.

The rationale for rotating the stimuli $180^{\circ}$ (inversion) across repetitions stems from research from the change blindness literature showing that inversion - unlike a left-right orientation change - is easily detected by participants. Shore and Klein (2000) used the flicker paradigm developed by Rensink, O'Regan, and Clark (1997) to investigate change blindness. In the flicker paradigm, a naturalistic scene is presented for a brief time, followed by a blank screen, and then the original scene is represented with an item in the scene changed. The two versions of the scene flicker back and forth until the participant notices the change. In the Rensink et al. study, it was noted that changes to items of central interest were noticed more quickly than changes to more peripheral items. Shore and Klein followed up this finding by conducting a similar experiment in which the changed elements of the scene were inverted. In this case, an advantage for inverted items of central interest was not found; that is, both central and peripheral changes were detected equally often.

Other research has demonstrated that inversion creates a salient stimulus change. Klein (1982) found that inversion has more dramatic effects on participants' perception of a stimulus than do $180^{\circ}$ rotations of the same 
stimulus in different dimensions (i.e., rotation on the $y$ and $z$-axes). Rock (1974) also provided a demonstration of the saliency of inversion as a stimulus change when he showed participants pictures of famous faces and outlines of common geographical formations, such as the United States, in an inverted form and found that recognition was impaired. We chose to use the inversion manipulation of object orientation in this experiment in order to further test the role of orientation in the effect and to determine whether a more salient orientation change would attenuate the primacy effect in memory for repetition.

\section{Method}

Participants. Forty-five students from Binghamton University participated to receive partial credit toward the completion of a course requirement.

Design and Materials. The materials and procedures used were identical to those in Experiment 1, with the exception that objects were now inverted instead of mirror reversed. In addition, stimuli were selected so as to eliminate any ecologically invalid variations of the objects. For instance, pictures of a camel, a barn, and a candle were removed from the stimulus set, whereas pictures of a pencil, a book, and a pair of scissors were retained in the stimulus set.

\section{Results and Discussion}

A 3 (type: all same, first different, or last different) $\times$ 3 (response: majority, minority, or both) repeated measures ANOVA was used to analyze the data. A main effect of response was found $\left[F(2,88)=82.93, M S_{\mathrm{e}}=\right.$ $0.05]$, indicating that the participants were more likely to choose a both response. A type $\times$ response interaction was also found $\left[F(4,176)=29.05, M S_{\mathrm{e}}=0.02\right]$. The comparison of central interest in this experiment did not reveal any significant effect. In Experiment 5, there was a slight numeric trend toward a primacy effect; however, this trend did not approach significance $[t(44)=0.78$, $p>.43]$; the participants were no more likely to choose the first-presented minority version of a stimulus than they were to choose the last-presented minority version of the stimulus.

Looking time data for Experiment 5 were analyzed in a manner similar to that in the previous experiments. A one-way ANOVA analyzing presentation order was significant $\left[F(4,176)=33.46, M S_{\mathrm{e}}=64,796.30\right]$, reflecting the fact that the participants looked longer at items being presented for the first time. Pairwise comparisons revealed significant differences between each serial position except the second and third presentations and the third and fifth presentations. In general, the pattern of the looking time data was similar to those in the previous experiments.

Experiment 5 demonstrates that a primacy effect in memory for repetition will not occur for all orientation changes. This is consistent with research showing differences in the effect of object inversion and left-right orientation changes on object recognition and change blindness (Bartlett et al., 1987; Cooper et al., 1992; Shore $\&$ Klein, 2000). The results also provide evidence that the primacy effect in memory for repetitions is not as robust a finding as originally was anticipated. The results of Experiments 3 and 4 suggested that this may be the case but were inconclusive in that the lack of a significant effect could have been argued to be a result of lack of featural overlap between the repetitions. Because object features were not manipulated in Experiment 5, the reversal of the primacy effect in memory suggests that the effect may not be characteristic of all human interactions with repetitions; rather, the scope of the effect may be limited to changes in size and right-left orientation.

\section{GENERAL DISCUSSION}

DiGirolamo and Hintzman (1997) provided empirical evidence confirming the old saying that first impressions are lasting impressions. The purpose of the present study was to investigate the finding of a primacy effect in memory for repetitions. Specifically, we sought to determine the generality of the effect across different changes in the stimuli across repetitions. We also investigated the effect in a situation in which participants were given an encoding task that drew the participants' attention to the changed attributes of the repeated stimuli.

The results of Experiments 1 and 2 show that the primacy effect in repetitions is reliable when a mirror reversal manipulation is used to change the stimuli across repetitions. Because of the large number of study list presentations and the subtlety of the changes in the stimuli, one could hypothesize that the participants were processing the stimuli in a cursory manner that would not include an encoding of the stimulus features that were manipulated across presentations. However, the results of Experiment 2 suggest otherwise. The primacy effect persisted when the participants made judgments about the left-right orientation of each object on the study list - a manipulation that was designed to ensure that the participants attended to the characteristic that changed across repetitions.

The present results also demonstrate some important boundary conditions for the effect. In Experiment 3, a subtle change in the features of objects did not result in a primacy effect for the first version of the object, and in Experiment 4, a color change across repetitions did not result in a bias toward the first presentation of the object. Rather, when two versions of an object were shown and the participants claimed to have seen only one version, there was no difference in the probability of choosing the first and the last versions of the object. Indeed, when the stimulus change was an inversion, the primacy effect was also attenuated. The results suggest that the primacy effect in memory for repetitions is not general across all stimulus changes; rather, it may occur in a limited number of circumstances.

The circumstances in which the primacy effect in memory for repeated stimuli does occur are informative. As was demonstrated by DiGirolamo and Hintzman (1997), the effect occurs when the reflection (normal vs. mirror reversed) and the size of the objects are changed across 
presentations. Experiments 1 and 2 in the present article replicate the effect with mirror reversals. However, we found no significant primacy effect with feature changes, color changes, or stimulus inversion. This pattern of results dovetails nicely with findings from the object recognition literature that demonstrate that so-called accidental properties (e.g., Roediger \& Srinivas, 1993) of stimuli, such as the right-left orientation and size, are represented differently in memory than are other characteristics.

The results of the present study join those in numerous studies in the object recognition literature that show that stimulus properties such as size and left-right orientation are represented differently than some other object characteristics. For example, Ellis et al. (1989) found that participants were significantly faster to name pictures of the same object rotated to a different angle than to name different examples of objects with the same name and that this advantage is relatively long lasting. This led Ellis et al. to hypothesize two visual codes, the first being a short-lived size- and viewpoint-specific code, and the second being a size- and viewpoint-invariant object representation that develops slowly but is longer lasting. The present results are consistent with these findings, since the study list was long enough that one would expect that there would be sufficient time for viewpoint-invariant representations of the objects to be developed.

In general, the results of these experiments add to the growing body of literature that points toward a memory system that is quite vulnerable to confusion regarding specific types of orientation changes across time. The present findings support previous studies by Bartlett et al. (1987) and Kanwisher et al. (1999), which provide evidence for poor recognition of changes to object orientation. Indeed, the present results also show that the primacy effect in memory for repetitions is not robust across conditions that do not rely on the accidental properties (Roediger \& Srinivas, 1993) of the stimuli presented.

One of the primary explanations of the primacy effect in memory for repetitions offered by DiGirolamo and Hintzman (1997) was simply that more attention is paid to the first presentation of a stimulus. There is much evidence to support this claim. Evidence from ERP studies has shown that the ERPs of repeated presentations are significantly different from those of first presentations of line drawings of objects (Rugg et al., 1995). PET studies have shown that cortical and subcortical areas in the temporal lobes are more active when participants view novel stimuli than when viewing a similar but familiar stimulus (Tulving et al., 1994). Eye-tracking technology tells us that when participants are exposed to repeated presentations of a scene, the participants make fewer fixations and sample fewer regions of the stimulus (Althoff $\&$ Cohen, 1999). Indeed, study time data from these experiments show that participants look longer at the first presentation of an object than at subsequent presentations. However, it must be noted that although this pattern occurred in all five experiments, the primacy effect in memory for repetitions did not; this suggests that the extra attention to the first presentation does not always translate into a memory bias.

The results of the experiments reported here provide some support for an account of the primacy effect in memory for repetitions in which the cognitive system does not represent/encode the changed attribute of the stimulus. Changing the accidental properties of stimuli across repetitions in the study list may lead participants to reactivate the previously studied versions of the objects when they reencounter mirror-reversed versions of the stimuli, rather than to form separate representations of the objects in their mirror-reversed forms. This account of the primacy effect in memory for repetitions is consistent with previous findings from the registrationwithout-learning literature, in which repetitions are registered but do not lead to an increased ability to discriminate between similar stimuli (Hintzman \& Curran, 1995; Hintzman et al., 1992). This view is also consistent with evidence that mirror-reversed stimuli and other manipulations of the accidental properties of the stimuli appear to be special cases of stimuli manipulations. Indeed, the results of the experiments reported here indicate that changes to the accidental properties of the stimuli across repetitions during the study list may be the only stimulus changes that result in a primacy effect in memory for repetitions.

In summary, the present research allows us to draw two broad conclusions. The first of these is that the primacy effect in memory for repetitions is replicable and reliable when stimulus changes involve the accidental properties of the stimulus (Experiments 1 and 2). Indeed, the results of these experiments build on the evidence first presented by DiGirolamo and Hintzman (1997) that there is a grain of truth behind the well-known colloquialism "first impressions are lasting impressions." However, a second conclusion that can be drawn from the reported experiments is that the primacy effect in memory for repetitions may not be robust to changes to stimuli that are not restricted to the accidental properties of the stimulus (Experiments 3-5). We see this second result as good news for those among us who have made a bad impression on a first date or job interview or have made an error when setting a VCR for the first time, since first impressions do not necessarily last as long as we might have thought.

\section{REFERENCES}

Althoff, R. R., \& Cohen, N. J. (1999). Eye-movement-based memory effect: A reprocessing effect in face perception. Journal of Experimental Psychology: Learning, Memory, \& Cognition, 25, 997-1010.

Bartlett, J. C., Gernsbacher, M. A., \& Till, R. E. (1987). Remembering left-right orientation of pictures. Journal of Experimental Psychology: Learning, Memory, \& Cognition, 13, 27-35.

Biederman, I., \& Gerhardstein, P. C. (1993). Recognizing depthrotated objects: Evidence and conditions for three-dimensional viewpoint invariance. Journal of Experimental Psychology: Human Perception \& Performance, 19, 1162-1182.

Cooper, L. A., Schacter, D. L., Ballesteros, S., \& Moore, C. (1992). Priming and recognition of transformed three-dimensional objects: Effects of size and reflection. Journal of Experimental Psychology: Learning, Memory, \& Cognition, 18, 43-57. 
Crowder, R. G. (1976). Principles of learning and memory. Hillsdale, NJ: Erlbaum.

DiGirolamo, G. J., \& Hintzman, D. L. (1997). First impressions are lasting impressions: A primacy effect in memory for repetitions. Psychonomic Bulletin \& Review, 4, 121-124.

Dunlosky, J., \& Thiede, K. W. (1998). What makes people study more? An evaluation of the factors that affect self-paced study. Acta Psychologica, 98, 37-56.

EbBINGHaUs, H. (1964). Memory: A contribution to experimental psychology. New York: Dover. (Original work published 1885)

Ellis, R., Allport, D. A., Humphreys, G. W., \& Collis, J. (1989). Varieties of object constancy. Quarterly Journal of Experimental Psychology, 41A, 775-796.

Greene, R. L. (1992). Human memory: Paradigms and paradoxes. Hillsdale, NJ: Erlbaum.

Hintzman, D. L., \& CurRan, T. (1995). When encoding fails: Instructions, feedback, and registration without learning. Memory \& Cognition, 23, 213-226.

HinTZMan, D. L., Curran, T., \& OpPy, B. (1992). Effects of similarity and repetition on memory: Registration without learning. Journal of Experimental Psychology: Learning, Memory, \& Cognition, 18, 667-680.

KANWISHER, N., YIN, C., \& WoJCIULIK, E. (1999). Repetition blindness for pictures. In V. Coltheart (Ed.), Fleeting memories: The cognition of brief visual stimuli (pp. 119-150). Cambridge, MA: MIT Press.

KLEIN, R. (1982). Patterns of perceived similarity cannot be generalized from long to short exposure durations and vice versa. Perception \& Psychophysics, 32, 15-18.

Levin, D. T., Simons, D. J., Angelone, B. L., \& Chabris, C. F. (2002). Memory for centrally attended changing objects in an incidental realworld change detection paradigm. British Journal of Psychology, $\underline{\mathbf{9 3}}_{2}$ 289-302.

MADIGAN, S., \& Rouse, M. (1974). Picture memory and visual generation processes. American Journal of Psychology, 87, 151-158.

NiCKerson, R. S., \& ADAMS, M. J. (1979). Long term memory for a common object. Cognitive Psychology, 11, 287-307.

O'Regan, J. K., Rensink, R. A., \& Clark, J. J. (1999). Change blindness as a result of "mudsplashes." Nature, 398, 34.

RaO, K. V., \& Proctor, R. W. (1984). Study-phase processing and the word frequency effect in recognition memory. Journal of Experimental Psychology: Learning, Memory, \& Cognition, 10, 386-394.

RENSINK, R. A., O'REgan, J. K., \& ClaRK, J. J. (1997). To see or not to see: The need for attention to perceive changes in scenes. Psychological Science, 8, 368-373.

Rock, I. (1974). The perception of disoriented figures. Scientific American, 230, 78-85.

ROEDIGER, H. L., III, \& SRINIVAS, K. (1993). Specificity of operations in perceptual priming. In P. Graf \& M. E. J. Masson (Eds.), Implicit memory: New directions in cognition, development, and neuropsychology (pp. 17-44). Hillsdale, NJ: Erlbaum.

RugG, M. D., Soardi, M., \& Doyle, M. C. (1995). Modulation of event-related potentials by the repetition of drawings of novel objects. Cognitive Brain Research,, 3, 17-24.
Schacter, D. L., Cooper, L. A., \& Delaney, S. M. (1990). Implicit memory for unfamiliar objects depends on access to structural descriptions. Journal of Experimental Psychology: General, 119, 5-24.

Shaughnessy, J. J., Zimmerman, J., \& Underwood, B. J. (1972). Further evidence for the MD-DP effect in free recall learning. Journal of Verbal Learning \& Verbal Behavior, 11, 1-12.

Shore, D. I., \& KLEIN, R. M. (2000). The effects of scene inversion on change blindness. Journal of General Psychology, 127, 27-43.

Simons, D. J. (2000). Current approaches to change blindness. Visual Cognition, 7, 1-15.

SimONS, D. J., \& LEVIN, D. T. (1998). Failure to detect changes to people during a real-world interaction. Psychonomic Bulletin \& Review, 5, 644-649.

SNODGrass, J. G., \& VAnderwart, M. (1980). A standardized set of 260 pictures: Norms for name agreement, image agreement, familiarity, and visual complexity. Journal of Experimental Psychology: Human Learning \& Memory, 6, 174-215.

SRINIVAS, K. (1995). Representation of rotated objects in explicit and implicit memory. Journal of Experimental Psychology: Learning, Memory, \& Cognition, 21, 1019-1036.

SRINIVAS, K. (1996). Size and reflection effects in priming: A test of transfer-appropriate processing. Memory \& Cognition, 24, 441-452.

Tulving, E., Markowitsch, H. J., Kapur, S., Habib, R., \& Houle, S. (1994). Novelty encoding networks in the human brain: Positron emission tomography data. NeuroReport, 5, 2525-2528.

Zimmerman, J. (1975). Free recall after self-paced study: A test of the attention explanation of the spacing effect. American Journal of Psychology, 88, 277-291.

\section{NOTE}

1. The looking time data from Experiment 1 were further subjected to a post hoc analysis by sorting the data by participant accuracy and then examining the looking times in the first-different condition. For each participant, response times were separated on the basis of whether the participant had responded correctly to that item on the ensuing recognition memory test. This analysis was performed because overall accuracy was less than optimal in these experiments and it may have been that the participants' looking times reflected differences among the experimental conditions for items that they got correct. For example, it is easy to envision participants' pausing to take a closer look at an item that they noticed had changed orientations from past exposures. If this were the case, we would expect looking times for items in the first-different condition that the participants got correct to be longer in Segment 2 (because the participants noticed the change). A repeated measures $t$ test was performed comparing the means of these two conditions, and no difference was observed $[t(29)=1.41, S E M=55.89]$. So the results of this analysis suggest that looking times did not vary according to the accuracy of the response.

(Manuscript received May 20, 2003; revision accepted for publication April 1, 2004.) 